\title{
MODELO DEL PROCESO DE LOGÍSTICA EXTERNA DE LAS EMPRESAS LOCALIZADAS EN EL MUNICIPIO DE FUNZA - CUNDINAMARCA
}

\section{EXTERNAL LOGISTICS PROCESS MODEL FOR COMMERCE COMPANIES OF THE MUNICIPALITY OF FUNZA - CUNDINAMARCA}

\begin{abstract}
Jairo Vásquez Bernal ${ }^{1}$; Pedro Layton ${ }^{2}$
${ }^{1}$ Mercadotecnista, Esp Gestión Social y Ambiental. Docente Investigador. Facultad Ing. Comercial, Universidad de Ciencias Aplicadas y Ambientales U.D.C.A; actualmente Corporación Tecnológica de Bogotá. Centro de desarrollo Empresarial, jairovasquez@ctb.edu.co; ${ }^{2}$ Ing. Comercial. Especialista en Finanzas. Docente Investigador, Facultad Ing. Comercial, Universidad de Ciencias Aplicadas y Ambientales U.D.C.A; pilaytonc@hotmail.com; dirección para correspondencia: Calle 56 No 17-15. Bogotá - Colombia.
\end{abstract}

Rev. U.D.C.A Act. \& Div. Cient. 16(2): 563-570, Julio-Diciembre, 2013

\section{RESUMEN}

La logística externa, como actividad empresarial, permite la administración de manera eficiente de los recursos, porque optimiza los procesos, reduce el gasto y aumenta la productividad. En su génesis, la logística solamente la aplicaban las fuerzas militares; actualmente, hace parte de los procesos comerciales, ya que se utiliza como herramienta fundamental para la operación y manejo de productos. El municipio de Funza es destacado en Cundinamarca por su dinámica comercial; sin embargo, no se ha estudiado el proceso particular de logística externa, de manera que se pueda contar con datos para mejorar el proceso logístico, por esto, se realizó, en primera instancia, una revisión bibliográfica y, posteriormente observaciones y entrevistas con informantes claves, a un grupo representativo de la población, muestra que se estableció por la distribución muestral de las medias y la selección de las unidades objeto de estudio, por el proceso de muestreo estratificado no proporcional. En este artículo, se presentan los resultados más relevantes de la investigación diagnóstica, en la que se destacan varios datos sobre el proceso de la logística externa en Funza. A partir de estos datos, se realizó un modelo que representa el proceso de la logística externa, fundamentado en modelos de regresión lineal simple y pruebas de correlación.

Palabras clave: Logística, transporte, proceso comercial, manejo de productos empresa.

\section{SUMMARY}

External logistics as a business activity allows managing resources efficiently by optimizing processes, reducing costs and increasing productivity. In its genesis logistics applied only to the military forces, now it is part of the business process, since it is used as an essential tool for the operation and management of products. Funza township is featured in Cundinamarca for its commercial dynamics, however, its particular external logistics process has not been studied, in order to dispose of the necessary data to improve the logistics process; therefore, in the first instance, a literature review was organized, followed by observations and key informant interviews with a representative population group, established by a sample distribution of the means and the selection of the units studied by the process of not proportional stratified sampling. This paper presents the most relevant results of the diagnostic research that highlights various data on the process of external logistics of the town of Funza. From these data, a model was accomplished that represents the external logistics process based on a simple linear regression and correlation tests model.

Key words: Logistics, transportation, business process management products, company. 


\section{INTRODUCCIÓN}

El sistema comercial incluye una estructura para cumplir los objetivos estratégicos y operativos de la empresa, igualmente, permite el diseño y la definición de las estrategias comerciales, necesarias para construir ventajas competitivas o mejorar las comparativas. Como lo expresan López-Pinto et al. (2010), el sistema de comercialización contempla, por una parte, la demanda de los consumidores y, por otra, los productos o servicios que las empresas ponen a disposición del comprador o del mercado. También se aplica para hacer que los productos se ubiquen en el momento y lugar que los consumidores los deseen (Lamb et al. 2006).

La palabra logística, etimológicamente procede de la palabra griega logist, que se define como flujo de materiales y es en la actividad militar, donde empieza a aplicarse (Anaya, 2007). En las empresas, comienza a tener relevancia en la década del sesenta y es entendida como el conjunto de técnicas y medios destinados a gestionar el flujo de materiales y de información, con el objetivo principal de lograr la satisfacción de necesidades en bienes y servicios de un cliente o un mercado, en cantidad, en calidad, en lugar y en momento (Urzelal, 2006).

Soret Los Santos (2006) define logística, como la parte del proceso de gestión de la cadena de suministro encargada de planificar, de implementar y de controlar, de forma eficiente y efectiva, el almacenaje y el flujo directo e inverso de los bienes, servicios y toda la información relacionada con estos, entre el punto de origen y el punto de consumo o demanda, con el propósito de cumplir con las expectativas del consumidor.

Según Casanovas \& Cuatrecasas (2012), en el proceso logístico, se planifica, se controla y se implementa, de manera eficiente y eficaz, el flujo y el almacenamiento de materia prima, de productos en proceso y de productos terminados, desde el punto de origen hasta el punto de consumo, a fin de satisfacer las necesidades de los clientes.

Precisamente, la diversidad de actividades que integran la logística y la operación de las mismas, ha acuñado otros elementos, como el transporte, el almacenamiento y los sistemas de información, que pareciera fraccionan el proceso general en otros subprocesos, pero, a su vez, son entendidos como un todo. Esta particularidad ha hecho que la logística, en la actualidad, se entienda como un proceso integral, en donde las actividades externas e internas, se estudian de manera particular, no importa que las mismas exijan relacionarse e, incluso, integrarse en su operación.

Para Urzelai (2006), la logística externa relaciona los cambios tecnológicos, la calidad de la competencia y la diversificación de los mercados. Afirma, igualmente, que la logística está llamada a tener un papel central en el seno de las empresas y en los circuitos físicos de los productos, porque controla hasta el $30 \%$ de los costos de la empresa.

Como lo sugiere Peris (2006), el problema al que se enfrentan muchas empresas es que no mueven suficiente volumen de productos y de materias primas, como para garantizar transportes rentables en el momento requerido por los empresarios, clientes o consumidores, los que les exigen entregas en lotes muy pequeños, con una gran frecuencia; la solución es integrar con otros operadores, pequeñas cargas para completar un tráiler y rentabilizar el transporte de larga distancia, función de la logística externa.

Esto hace que, cada día, sea necesario acudir a herramientas tecnológicas especializadas, para prospectar en escenarios diversos, diferentes alternativas y acercarse a un suceso con menos incertidumbre (Piera et al. 2006). Las técnicas de simulación, se emplean para predecir el comportamiento de sistemas complejos y para comprender los impactos del cambio; permiten diagnosticar el sistema antes de ser construido; reducen el riesgo y el tiempo necesario de puesta en marcha o modificación de un sistema, como el relacionado con la logística, en el municipio de Funza.

Funza está ubicada en la provincia de sabana de Occidente, a $15 \mathrm{~km}$ de la ciudad de Bogotá. Limita al Norte, con los municipios de Madrid y de Tenjo; al Oriente, con los municipios de Cota y Bogotá, D.C.; al Sur, con el municipio de Mosquera y al Occidente, con el municipio de Madrid. Tiene una extensión urbana de $4 \mathrm{~km}^{2}$ y una extensión rural de $66 \mathrm{~km}^{2}$, para un total de $70 \mathrm{~km}^{2}$. La superficie del municipio es plana, presentando las características típicas de la altiplanicie cundinamarquesa.

La base económica del municipio de Funza está conformada por el comercio $y$, especialmente, por las industrias manufactureras, cuya cantidad aumenta constantemente, debido al cambio en el uso del suelo establecido en el Plan de Ordenamiento Territorial -POT-, a los bajos impuestos y bajos costos de los servicios públicos y, adicionalmente, por su ubicación estratégica, cerca a la capital del país, que posibilita la entrada y la salida de mercancías desde y hacia la mayoría del territorio Colombiano.

\section{MATERIALES Y MÉTODOS}

Se consultaron fuentes de información primaria y secundaria, que permitieron identificar las características de las empresas del municipio de Funza, en cuanto a su actividad económica, perfil, tipo de organización y el manejo de los procesos de logística externa. En la revisión bibliográfica especializada, 
se encontraron informes sobre logística, elaborados por empresas e instituciones del orden nacional y municipal.

La investigación es descriptiva y analítica, ya que comprende el registro, el análisis y la interpretación de la naturaleza de los fenómenos (Tamayo, 2005), presenta la situación conceptual de los procesos empresariales y de las características de los sistemas de distribución y logística empleados. Es analítica, porque se proponen constructos teóricos, a partir de la abstracción de los elementos y variables del sistema logístico, para diseñar y reformular procesos (Obradors, 2007).

El modelo de logística externa que se plantea relaciona las variables identificadas en el proceso logística que actualmente opera y el impacto de las mismas, en la permanencia de los automotores en el área urbana del municipio y en los parques empresariales, como capacidad de prever el futuro (Petri \& Gorven, 2006).

La obtención de la información primaria, se realizó a través de una muestra representativa de la población, la cual, se halló por la distribución muestral de las medias (Mas, 2010; Grande \& Abascal, 2009; Mcdaniel, 2007; Jany, 2005), cuya fórmula es:

$\mathrm{n}=\sigma \mathrm{X}^{2} \mathrm{Z}^{2} \mathrm{~N} / \mathrm{E}^{2}(\mathrm{~N}-1)+\sigma \mathrm{X}^{2} \mathrm{Z}^{2}$

$\mathrm{n}=$ Tamaño de la muestra

$\sigma \mathrm{X}^{2}=$ Varianza muestral

$\mathrm{E}=$ Error

$\mathrm{Z}=$ Intervalo de confianza

$\mathrm{N}=$ Tamaño de la población

La población es de 105 empresas, la desviación estándar poblacional de 6,3832667 , la media de la población es de 6,5625, la media de una muestra 4,375, el error es de 2,1875 , el intervalo de confianza 1,96. Con estos datos, se aplicó la fórmula para población finita, lo cual, da como resultado que las unidades empresariales para el estudio sean de 26 empresas.

Por las características de ubicación de las empresas en diferentes parques industriales, se hizo un muestreo estratificado, con base en la varianza estimada dada por el promedio de parques industriales así: pequeños, hasta 5 empresas; medianos, hasta 11 y grandes, más de 12 .

La obtención de información primaria de la logística externa, se realizó por muestreo estratificado no proporcional (Grande \& Abascal, 2009; Anderson et al. 2008; Zilmund, 2008; Marshall, 1999), mediante la técnica de observación en ocho puntos estratégicos, de los cuales, seis corresponden a los siguientes parques industriales: Argelia, El Hato, Galicia, San Antonio, La cofradía y San Carlos y dos áreas de empresas independientes: El Hato y km 9.
El estudio permitió la caracterización de las empresas y del proceso de logística de las organizaciones que funcionan en la zona industrial del municipio de Funza; igualmente, ayudó a establecer los elementos de la operación logística, que se requiere para la adopción del modelo.

El análisis y la interpretación de la información, se desarrolló con los resultados de la tabulación; se construyeron las tablas y las figuras; se establecieron sus interacciones, de tal forma que permitieron identificar las propiedades y el funcionamiento de la logística externa de las empresas localizadas en el municipio de Funza, para finalmente, proponer la estructura del modelo de logística externa, adecuado a la situación de manejo de mercancías.

\section{RESULTADOS Y DISCUSIÓN}

El conocimiento de la logística externa es importante, por el impacto económico, social y de movilidad, especialmente, en áreas con alto desarrollo, como la que se presenta en el municipio de Funza - Cundinamarca. El resultado, partiendo del análisis de la información recopilada, es el siguiente:

En el municipio de Funza funcionan 13 parques industriales, que aglutinan el 65,71\% de las empresas; el 34,29\% restante, se ubica en tres áreas independientes. Según el sector económico donde desarrollan su actividad, el 53,33\% de las empresas ofrecen servicios; el $42,86 \%$ realizan actividades de manufactura y el restante $3,81 \%$, se dedica a realizar actividades del sector agropecuario.

En las empresas prestadoras de servicios, se encontraron 17 categorías diferentes. El 37,50\% ofrecen servicios de logística y transporte; el $10,71 \%$, en comercio de materias primas; el 8,93\%, comercializan productos para ferreterías; el 5,93\%, distribuye productos y alimentos para el hogar, es importante indicar que se hallan bodegas de almacenes Éxito, Carrefour y Coratiendas; también con el 5,93\%, se evidenciaron empresas que ofrecen servicios petroleros y, en menor porcentaje, se encuentran empresas que comercializan productos de aseo personal, vestuario, electrodomésticos, artes gráficas y de productos farmacéuticos.

En empresas manufactureras, se encontraron doce categorías, dentro de las cuales, se destacan las transformadoras de plásticos y sus derivados, en un $19,75 \%$, empresas de alimentos, como Fryto Lay®, Big Cola ${ }^{\circledR}$, Colanta ${ }^{\circledR}$ y Rica Fruta $\AA$, para un $13,04 \%$; con el $10,87 \%$, las fábricas de empaques y de envases; con el mismo porcentaje, las materias primas y de implementos para la construcción; finalmente, con menores porcentajes, fábricas de maquinaria, aseo, autopartes, de cosméticos y farmacéuticos. Del sector agropecuario, se registran cuatro fábricas dedicadas a la 
producción de alimentos concentrados para animales y de insumos para los cultivos de la región.

En el municipio de Funza, se encuentran empresas de todos los sectores económicos, las de servicios son más de la mitad, pero de estas, la mayoría se relacionan con el servicio de manejo físico de los productos, ya que por su cercanía con Bogotá, facilitan la entrada y la salida de mercancías, a menores costos y mayores espacios; también es importante indicar que hay multinacionales que fabrican, exportan e importan productos.

Los lugares de donde llegan las mercancías son diversos; se destacan: Barraquilla (10,85\%), Cartagena (9,5\%), Buenaventura (9,01\%), Santa Marta (8,56\%), Bogotá (8,44\%), Cali $(7,69 \%)$ Medellín (6,33\%) y Villavicencio (5,88\%). Con menores porcentajes, Cúcuta, Bucaramanga, Sogamoso, Pasto, Duitama, entre otros.

Es importante indicar que el transporte de carga es oneroso para la empresa, por el costo de los vehículos, los peajes y el valor de la gasolina; adicionalmente, el gasto que se genera por las operaciones de recibo y de despacho, la asignación de equipos y de personal, para llevar un control detallado de cada uno de los viajes realizados y, en general, cada uno de los procesos que exija la operación.

La logística externa impacta financieramente a la firma, pero adicionalmente, se genera mayor flujo vehicular al municipio, ocasionando problemas de movilidad, trancones por la demora en carga y descarga dentro del parque empresarial, en razón que los espacios para parqueo dentro de los mismos son pequeños y no pueden albergar el total de vehículos que le ingresan, generando filas al lado de la vía.

Con relación a lo anterior, el estudio permitió establecer la frecuencia de entrada y de salida de vehículos por tamaño y por capacidad, durante los días hábiles de la semana, como se establece en la tabla 1 . Se destaca que durante la semana, el martes se concentra la mayor cantidad de camiones y corresponde, principalmente, a las mercancías que llegan de los puertos y representa casi el doble del lunes y el viernes. También se resalta que los camiones de dos ejes son los que representan mayor porcentaje y en relación con las tractomulas, la que circula más por el municipio es la de cabezote de tres ejes, con semirremolque de tres ejes, principalmente, por la capacidad de carga.

Otra actividad del proceso de logística externa es el descargue de los camiones, cuyo resultado global para el área de observación es el siguiente: Descargar un camión en promedio demora 1,99 horas, con la participación directa de 2,68 personas en promedio. La tractomula de tres ejes es la que en promedio exige más tiempo 2,53 horas y mayor cantidad de personas descargando, 3,75; el que se descarga más rápido es el camión de dos ejes pequeños, 0,62 horas.

Cargar un automotor exige, en promedio, 2,51 horas, superior en 0,52 horas a la descarga; el tipo de camión que más requiere tiempo para la carga es la tractomula de tres ejes, 3,09 horas y el número de personas para desarrollar la actividad es de 3,75; el camión de dos ejes pequeño, se

Tabla 1. Análisis de correlación para la carga.

\begin{tabular}{|c|c|c|c|c|c|}
\hline \multicolumn{6}{|c|}{ Correlaciones para carga } \\
\hline & Tiempo carga & Personas carga & $\begin{array}{c}\text { Número de ejes } \\
\text { camión }\end{array}$ & $\begin{array}{l}\text { Número de ejes } \\
\text { del remolque }\end{array}$ & Centro \\
\hline Tiempo carga & 1 &, $596^{* *}$ &, $266^{*}$ &, $508^{* *}$ & 0,166 \\
\hline Personas carga &, $596^{* *}$ & 1 & ,249* &, $646 * *$ & 0,123 \\
\hline $\begin{array}{c}\text { Número de ejes } \\
\text { camión }\end{array}$ & ,266 & ,249* & 1 & 0,087 & 0 \\
\hline $\begin{array}{l}\text { Número de ejes } \\
\text { del remolque }\end{array}$ &, $508^{* * *}$ &, $646^{* *}$ & 0,087 & 1 & 0 \\
\hline Centro & 0,166 & 0,123 & 0 & 0 & 1 \\
\hline \multicolumn{6}{|c|}{ **La correlación es significativa al nivel 0,01 (bilateral). } \\
\hline \multicolumn{6}{|c|}{ *La correlación es significante al nivel 0,05 (bilateral). } \\
\hline
\end{tabular}


carga en menor tiempo, 1,05 horas y el número personas requerido es de 1,25 .

El 9,39\% de los despachos, se realiza para Bogotá, por su cercanía y también porque es uno de los centros de consumo más grandes del país; también, se envían mercancías a otros lugares, como Cartagena (8,59\%), Cali (8,16\%), Cúcuta (8,16\%), Barranquilla (8,16\%), Medellín $(6,53 \%)$, Buenaventura $(5,71 \%) \mathrm{y}$, en menores porcentajes, a las ciudades capitales y a municipios cercanos a Bogotá, como Cota, Chía, Mosquera, Madrid y Cajicá.

Al comparar el proceso de logística es importante destacar al municipio de Cota, en Cundinamarca, ya que es en donde se encuentran parques industriales como: Celta Trade Park, Los Nogales, Metropolitano, Parque Industrial Siberia, con empresas como Marcopolo, fábrica de buses articulados tipo Transmilenio, Yanbal ${ }^{\circledR}$, Pat Primo $\AA$, Gerfar ${ }^{\circledR}$ y algunas empresas con infraestructura propia, como Colombina ${ }^{\circledR}$, industrias sprint, Copidrogas, Siemens y Titán.

En el municipio de Mosquera, se encuentra parques industriales como: San Jorge, Montana, Puerto Vallarta, Porvenir y cuenta con muchas empresas importantes, como
Ramo, Purina, Finca, Harinas El lobo, Doria, Soya, Fósforos El Rey, entre otras. Y en el municipio de Madrid, se está construyendo una zona franca y tres parques industriales; se destacan las sedes de Colcerámica S.A. (empresa de la organización Corona), Café Águila Roja ${ }^{\circledR}$, Ajover ${ }^{\circledR}$, Factoría del Vidrio Bellini ${ }^{\circledR}$, Molinos Capri ${ }^{\circledR}$, Facelec ${ }^{\circledR}$, Triplex y Acemar®.

Al comparar el proceso de logística externa de las empresas localizadas en Funza, con otras empresas de otros municipios, se observa que en los municipios cercanos a Bogotá, también se concentran empresas de diferentes actividades, aunque en todos los desarrollos son diferentes. El proceso logístico de Funza es el de más impacto por la cercanía a la capital colombiana, ya que se puede entrar por la calle $80 \mathrm{y}$ la calle 13 y por la facilidad de llegar al aeropuerto El Dorado de Bogotá.

La estructuración del modelo de logística externa, se realiza a través dos componentes principales, que son el tráfico y el tiempo de carga y descarga. El análisis del tráfico, se desarrolla utilizando los indicadores de intensidad, densidad y capacidad de la vía, los resultados de intensidad se presentan en la figura 1.

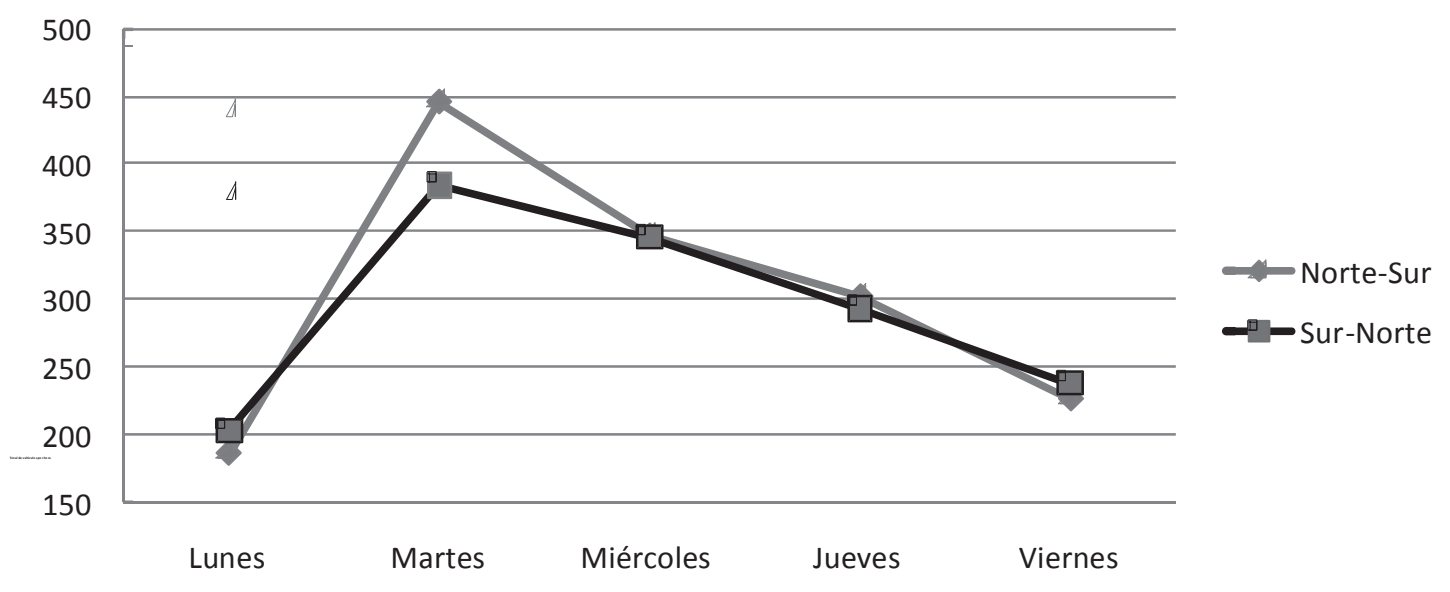

Figura 1. Intensidad de tráfico - hora.

Con base en los resultados, se determinó que la intensidad de tráfico es mayor el martes. En general, se encuentra que el flujo de camiones norte-sur es equivalente al sur-norte, a excepción del martes, que presenta un mayor tráfico de camiones en sentido norte-sur. Es importante indicar que la fórmula general para el cálculo de la densidad del tráfico es la siguiente:

Densidad $=$ Intensidad $/$ Velocidad media
Considerando la anterior ecuación y teniendo como base que la velocidad media de $60 \mathrm{~km} /$ hora, se obtiene la siguiente densidad de tráfico 39,64 vehículos/hora. La densidad del tráfico es mayor en el sentido sur-norte, lo que significa una menor fluidez de los camiones; también es importante indicar que el martes tiene la mayor intensidad de tráfico, 55,37 vehículos/hora.

Respecto al análisis de los tiempos de carga y de descarga, se fundamenta en los modelos de regresión lineal simple 
y validada con pruebas de correlación. A continuación, se presenta el resumen de las principales estadísticas.

Se encuentra que, en general, es mayor el tiempo de carga que el de descarga; sin embargo, el coeficiente de variación indica que es mucho más predecible el tiempo de carga $(\mathrm{CV}=38 \%)$ de que de descargue $(\mathrm{CV}=45 \%)$.

Del cálculo de los percentiles se resalta: El 25\% de las cargas realizadas toma dos horas o menos, mientras que las descargas tardan una hora y media, en el $25 \%$ de los casos; es importante indicar que el tiempo medio de descargue es menor al de carga, por 30 minutos y el $90 \%$ de las cargas realizadas, se tardó entre una y tres horas y media, mientras el $90 \%$ de los descargues tomó tres horas, como se observa en la figura 2, Box-plot tiempo de carga y descarga.
La figura 2, Box-plot permite constatar las afirmaciones ya realizadas y como información relevante para el modelo de predicción del tiempo de carga y de descargue, se tiene que no se encuentran observaciones atípicas. Al observar las estadísticas descriptivas para las variables número de personas que se emplean en la carga y el número de personas que se emplean en el descargue, se encuentra que no presentan diferencias significativas, destacando que el $90 \%$ de las cargas las realizan cuatro personas, $75 \%$ de las cargas, tres personas y el $25 \%$, dos personas.

El uso de Box-plot validó estas afirmaciones, además se estableció que el tiempo requerido para la carga de los camiones es superior al tiempo de descarga en 30 minutos promedio; el número de personas que se ocupa de la carga y la descarga de lo camiones es el mismo. En términos de

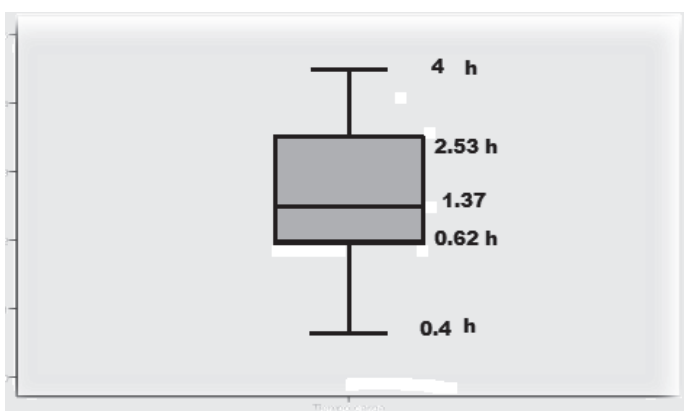

Box-plot tiempo de carga

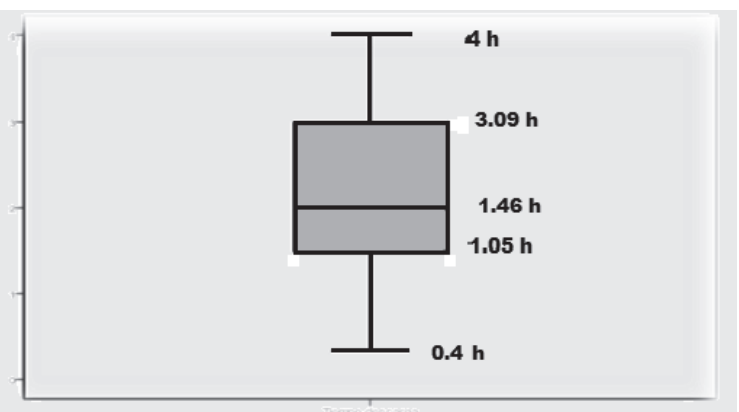

Box-plot tiempo de descarga

Figura 2. Box-plot tiempo de carga y de descarga.

la logística, las variables no inciden favorablemente en la reducción del tiempo de cargue y descarga, además, no se encuentran observaciones atípicas.

Existe relación directa entre el número de personas que intervienen en la carga o descarga y el tiempo que estas tardan; el número de ejes del remolque está relacionado directamente con el tiempo de carga o descarga, lo que significa que a mayor número de ejes del camión, el tiempo de carga y descarga es mayor; luego, existe relación directa entre el número de personas que interviene en el proceso y el número de ejes del remolque, como se observa en la figura 3 .

Finalmente, la capacidad es el valor máximo que adquiere la densidad, representada por la intensidad y la velocidad, lo que se conoce como "densidad crítica" y que se sintetiza en la figura 4, correspondiente al modelo de logística externa. Teniendo en cuenta los anteriores resultados y considerando la definición de Highway Capacity Manual, para la vía de análisis se obtienen los siguientes resultados de capacidad: la utilizada del sentido Norte-Sur: 446 camiones por hora y la capacidad del sentido Sur- Norte: 384 camiones por hora, con lo cual, se establece que el promedio por hora se encuentran 415 camiones, valor medio de la capacidad total, a partir, del cual, el espacio disponible se satura.

Conflicto de intereses: El manuscrito fue preparado y revisado con la participación de todos los autores, quienes declaramos que no existe ningún conflicto de intereses, que ponga en riesgo la validez de los resultados presentados. Financiación: Este estudio fue financiado por la U.D.C.A y la Alcaldía de Funza. 


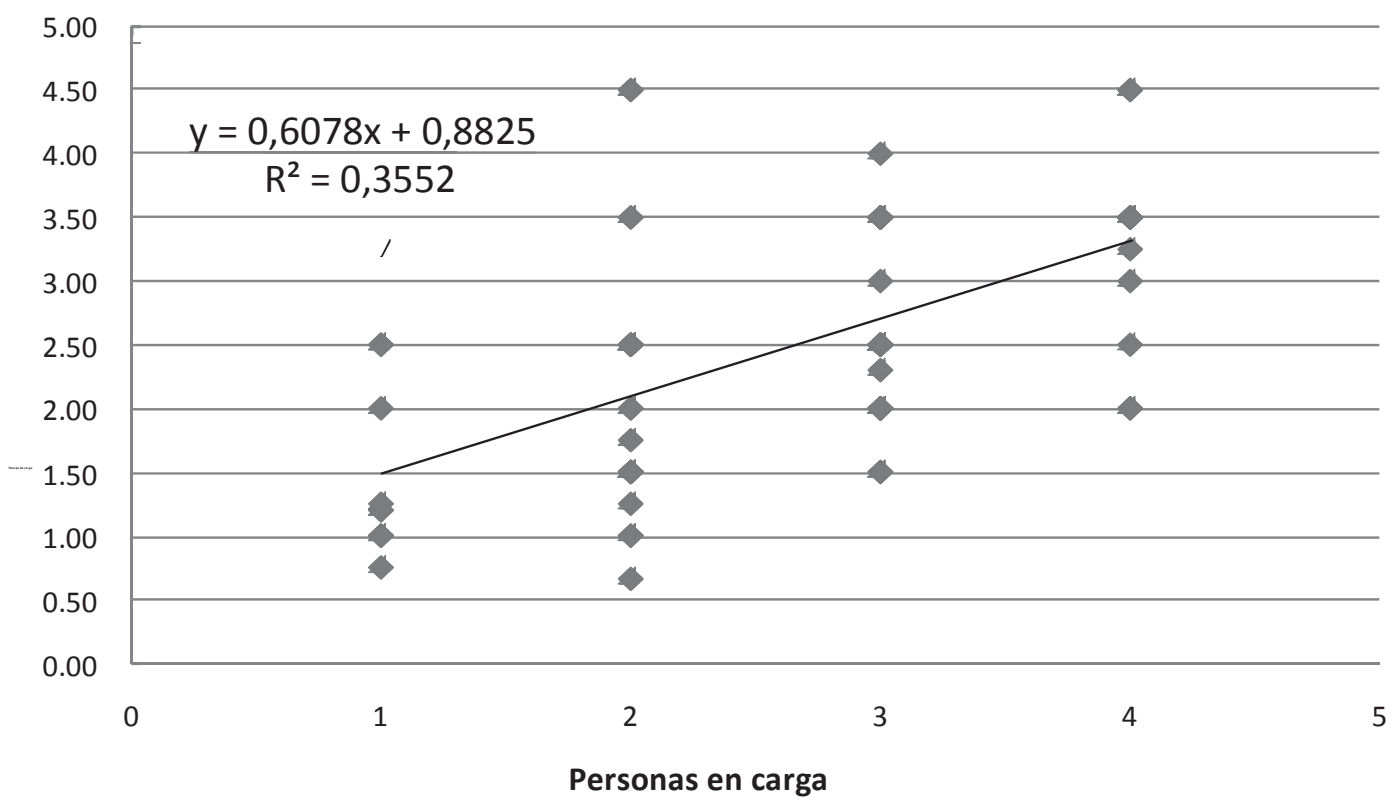

Figura 3. Relación tiempo - personas en carga.

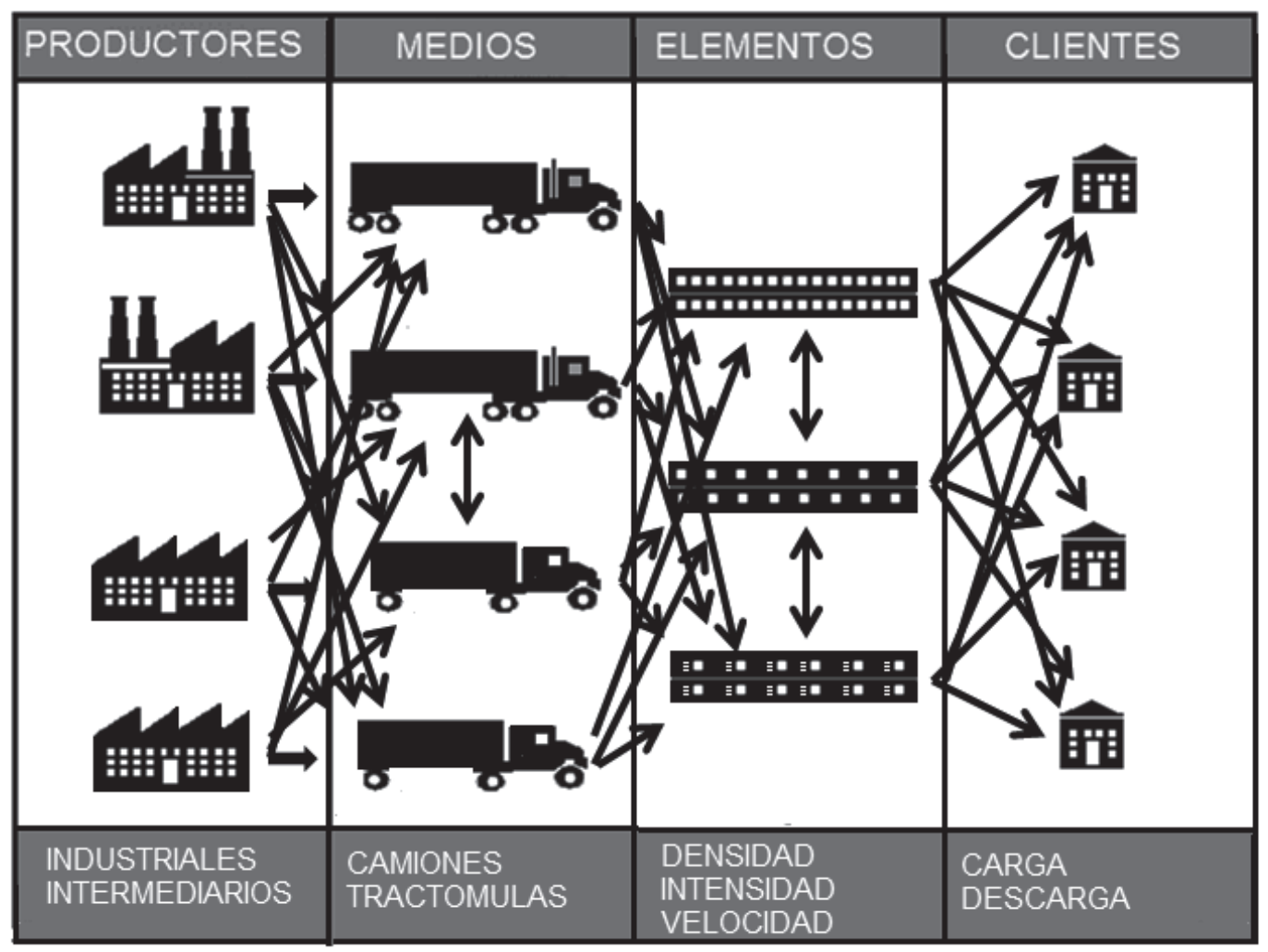

Figura 4. Modelo de logística externa. 


\section{BIBLIOGRAFÍA}

1. ANAYA, J. 2007. Logística integral: la gestión operativa de la empresa. $3^{\text {ra }}$ ed. Editor ESIC Editorial (España). 290p.

2. ANDERSON, D.; SWEENEY, D.; WILLIAMS, T. 2008. Estadística Para Administración y Economía. Cengage Learning Editores (México). 1056p.

3. CASANOVAS, A.; CUATRECASAS, L. 2012. Logística Integral. Nuevas Tendencias en logística y Operaciones. Profit Ed. (España). 214p.

4. GRANDE, I.; ABASCAL, E. 2009. Fundamentos y Técnicas de Investigación Comercial. Esic Editorial (España) 433p.

5. JANY, N. 2005 Investigación Integral de Mercados: Un enfoque para el siglo 21. 3ra ed. Ed. Mc Graw Hill (Santafé de Bogotá) D.C. 409p.

6. LAMB, W.; HAIR, J.; MCDANIE, C. 2006. Marketing. 8va ed. Cengage Learning Editores. (México). 697p.

7. LÓPEZ-PINTO, B.; MACHUCA, M.; VISCARRI, J. 2010, Los Pilares del Marketing. Editorial de la Universidad Politécnico de Cataluña. (Barcelona).413p.

8. MARSHALL, K.P. 1999. Sistemas de Información de Mercadotecnia. International Thomson Editores. (México). 164p.

9. MAS, F. 2010 Temas de Investigación Comercial. 5 Ed. Editorial Club Universitario ECU. España. 496p.

10. MCDANIEL, C; 2007. Investigación de Mercados. $6^{\text {ta }}$ ed. México, Thomson. 617p.
11. OBRADORS, M. 2007. Creatividad y generación de ideas: Estudio de la práctica creativa en cine y publicidad. Universidad de Valencia. (España). 238p.

12. PERIS, S. 2006. Distribución comercial. 5ta Ed. Editorial ESIC. (España) 366p.

13. PETRI, H.; GORVEN, J. 2006. Motivación: Teoría, investigación y aplicaciones. Cengage Learning Editores (México). 504p.

14. PIERA, M.; GUASCH, T.; CASANOVAS, J.; RAMOS, J. 2006. Como mejorar la logística de su empresa mediante la simulación. Ediciones Díaz de Santos. (Madrid - España). 103p.

15. SORET LOS SANTOS, I. 2006. Logística y marketing para la distribución comercial. $3^{\text {ra }}$ Ed. ESIC Editorial. (España) 332p.

16. TAMAYO, M. 2005. El Proceso de la investigación científica: Incluye evaluación y administración de proyectos de investigación. Editorial Limusa (México). 440p.

17. URZELAI, I. 2006. Manual básico de la logística integral. Ediciones Díaz de Santos. Madrid (España). 320p.

18. ZILMUND, W. 2008. Investigación de mercados. $9^{\text {na }}$ ed. Cengage Learning Editores (México). 736p.

Recibido: Julio 4 de 2012

Aceptado: Octubre 30 de 2013

Como citar:

Vásquez Bernal, J.; Layton, P. 2013. Modelo del proceso de logística externa de las empresas localizadas en el municipio de Funza - Cundinamarca. Rev. U.D.C.A Act \& Div. Cient. 16(2): 563-570. 